\title{
Uncertain Optimization of Discrete Supply Networks with Order Delivery Disruption and Risk Preference in the Postepidemic Era
}

\author{
Yang Song $\mathbb{D}^{1,2}$ Yan-qiu Liu, ${ }^{1}$ Qi Sun $\mathbb{D D}^{3,4}$ Hai-tao Xu ${ }^{1 D},{ }^{5}$ and Ming-fei Chen $\mathbb{D}^{6}$ \\ ${ }^{1}$ School of Management, Shenyang University of Technology, Shenyang, China \\ ${ }^{2}$ School of Economics and Management, Liaoning Finance Vocational College, Shenyang, China \\ ${ }^{3}$ Antai College of Economics \& Management, Shanghai Jiao Tong University, Shanghai, China \\ ${ }^{4}$ Zhejiang Ribon Intelligent Equipment Co., Ltd., Jiaxing, China \\ ${ }^{5}$ Department of Electrical Engineering and Computer Science, Cleveland State University, Cleveland, OH, USA \\ ${ }^{6}$ SLZY (Shenyang) Hi-Tech Co., Ltd., Shenyang, China
}

Correspondence should be addressed to Yang Song; fiona-sy@outlook.com

Received 30 July 2021; Accepted 4 October 2021; Published 21 October 2021

Academic Editor: Baogui Xin

Copyright (๑) 2021 Yang Song et al. This is an open access article distributed under the Creative Commons Attribution License, which permits unrestricted use, distribution, and reproduction in any medium, provided the original work is properly cited.

Epidemic blockade leads to increased uncertainty and dynamic supply network disruption. This study considers an uncertain optimization of dynamic supply networks with risk preference and order delivery disruption. Taking the subjective utility of downstream enterprises as a reference point for the utility measurement of order delivery disruption and risk preference, this study constructs a biobjective optimization model with the goal of maximizing the downstream firm's subjective utility and minimizing the manufacturer's cost. The influence of each parameter in the downstream firm's subjective utility function on the integrated optimization was analysed. The research found that the uncertain optimization model with the risk preference of downstream firms for order delivery disruption better controls the actual manufacturer's order allocation and distribution problems when considering the downstream firms' behaviour preference characteristics under bounded rationality. When allocating orders, manufacturers should consider that changes in order delivery disruption will cause changes in the subjective utility of downstream enterprises. In the process of multiperiod cooperation between manufacturers and downstream firms, they can obtain downstream firm risk preferences through repeated investigations.

\section{Introduction}

1.1. Background. The COVID-19 epidemic in 2019 is a major public health emergency that presented rapid transmission, a wide infection range, and difficult prevention and control, resulting in serious health hazards and economic and property losses. In more than 60 countries with "closed cities" and "closed countries," restrictions in the epidemic caused supply chain disruptions, thus leading to significant negative effects on global supply networks. Authoritative international medical experts predict that it is almost impossible for the fourth wave of the COVID-19 epidemic in the world to end this summer. At present, countries have strengthened epidemic prevention and control measures, including border control, curfew closure, and strict personnel flow control. However, these measures have increased the risk of stagnation of international trade and rupture of the global supply chain, which have had a serious impact on world economic and social development. Therefore, it is necessary to build a dynamic supply chain network to deal with emergencies and reduce epidemic losses. In the normalization management of epidemic situations, precise prevention and control policies will lead to isolation restrictions at any time. Due to the epidemic blockade in Nanjing in July 2021, the production or destination place adopted the blockade policy in the epidemic area, and people, vehicles, or products faced epidemic isolation or restriction. The disruption risk led to order delivery disruptions or delays. Any node error in the supply chain may lead to dysfunctions in or disruptions to the supply 
networks. Solving the product supply problem under blockade disruption has become a research hotspot for government departments, scholars, and managers.

1.2. Motivation. The Delta variant of the virus destroyed the port order in Southeast Asia in 2021, resulting in near "suffocation." The western coast of the United States is affected by hurricanes, which leads to the paralysis of ports and prevents ships from entering. Cape Town, South Africa, was attacked by the Internet and left in chaos. Severe emergencies have caused frequent disruptions to the global supply chain, and the failure of rapid turnover of funds and materials has led to a large-scale disruption of enterprise operations, resulting in huge economic losses. Scholars and decision makers pay more attention to the uncertainty optimization problem of discrete networks, with the goal of mitigating the disruption and quickly reducing the loss.

In April 2020, the COVID-19 epidemic caused the failure of order delivery, and Amazon suspended new user orders. Deteriorating internal and external uncertainties increase the risk of supply chain disruption. Scholars have found that coordinated delivery strategies can significantly improve the disruption response ability of uncertain supply networks. Therefore, this study investigated the impact of order delivery disruption on the integrated optimization of enterprise location paths with the goal of maximizing the utility of supply chain participants through a coordinated delivery strategy.

Interruption risk has uncertain characteristics, and subjective behaviour preference is shown in the uncertain environment of personal behaviour. The subjective risk preference of downstream enterprises in uncertain environments directly affects the optimization objective, and the disruption risk attitude determines the supply chain antiinterrupt system strategy. This study measures the disruption risk preference behaviour of downstream enterprises and provides a reference for enterprise investment selection or order decision making.

Investors understand that investment is risky, and they also want to obtain the maximum return through investment. How should enterprises make decisions when facing the trade-off between risk and return? The utility value is the equilibrium point of the relationship between risk and income and represents the equilibrium index of risk and income. Therefore, this study uses a utility function to analyse the risk behaviour preference of enterprises to help enterprises absorb or reduce the loss caused by disruption with diversified income.

1.3. Literature. During the COVID-19 epidemic in April 2020, Amazon temporarily stopped accepting orders from new users, resulting in large-scale order delivery disruptions. Delivery disruption refers to the failure or delay of order delivery caused by various internal and external uncertainties in the process of product transportation or distribution, which reduces the overall performance of the supply networks [1-4]. For the order delivery disruption or delay problem caused by the COVID-19 epidemic, scholars have designed a disruption recovery strategy of supply systems with changing product types [5]; they found that location routing optimization strategies can significantly improve supply network performance under disruption risk [6], designed a reliable location model with spare facilities to respond to delivery disruptions [7], and analysed the relationship between order delivery disruption and system performance and then set disruption variables to design an optimization model of combinatorial location and inventory problems [8]. In an uncertain network with random delivery disruption, the optimization of combinatorial location routing and inventory problems with multiple products and cycles has been studied, and the authors suggested that decision makers adopt partial order delay strategies to improve the reliability of supply networks [9]. In the case of supplier delivery disruption, the impact of delivery disruption on supply system lead time was found to be random [10]. Order delivery disruption caused by uncertainty is one of the critical factors affecting the optimization of discrete network systems.

When the COVID-19 epidemic broke out in Wuhan in 2020, the epidemic area was blocked and supply chain participants actively cooperated to overcome the delay or disruption of product supply. Participants' risk preference affects the system's antidisruption decision and the optimization decision of the discrete network. There are three types of risk preference: risk aversion, risk seeking, and risk neutrality [11]. Lau and Lau studied wholesale price decisions of risk-averse manufacturers and order quantity decisions of risk-averse retailers and found that risk preference has an influence on the optimal decisions [12]. Agrawal and Seshadri compared the optimal models of risk-neutral and risk-averse retailers and found that uncertainty and risk preference affect pricing strategies and optimal inventory decisions [13]. Tsay found that risk preference can influence partner selection strategy and designed a mean-variance measure of risk value for risk-averse decision makers [14]. Xanthopoulos et al. studied the disruption risk of dualsource supply channels, analysed the preference characteristics of risk neutrality and risk aversion, and designed the uncertainty optimization of combinatorial routing and inventory problems [15]. Supply network participants always want to obtain better future utility from investment, and scholars have found that the utility function can reflect participants' risk preference. Moreover, the downside risk, mean variance, and utility function were used as risk measurement tools to realize the multiobjective optimization of supply networks based on the Pareto optimal concept of decision theory $[1,11]$. Risk preference is one of the important influencing variables of the multiobjective optimization of discrete supply networks [16-19]. Centralized decision method is a set of comprehensive analysis on multivariate information through data quantification, especially abnormal data inspection and emergency response to interruption [20-22]. At present, few relevant research studies have used utility to measure risk preference.

These research results provide a very effective theoretical and practical basis for supply network optimization under epidemic blockade. These supply network anti-interruption 
works in the literature mainly focus on the coordination mechanisms and vulnerability mechanisms. Previous studies have not focused on uncertain discrete network optimization problems from the perspective of order delivery disruption and risk preference. Compared with the above research work, this study considers risk preference, cost, and utility and solves the combinatorial optimization problem of location allocation, path, and risk in discrete networks based on system dynamics theory.

1.4. Contribution. In view of the supply chain network disruption caused by uncertainties, such as order delivery disruption, this study proposed a biobjective linear programming mathematical model to describe the integrated optimization problem of uncertain networks. The costutility function in this study reflected the dual objective of minimizing the manufacturer cost and maximizing the utility of downstream enterprises and solved the integrated optimization problem of decision-makers' location paths in discrete networks. In the existing results, subjective utility is rarely used as a reference point to measure the model and solution of the risk behaviour preference of downstream enterprises, and these research results make up for the blank.

The location routing inventory integrated optimization problem is an NP-hard problem, and identifying the exact solution is difficult. In this study, $\varepsilon$-constraint and compromise algorithms are designed. After simplifying the cost constraints, the dual-objective model is simplified as a single-objective model, and the dual-objective compromise algorithm is used to solve the optimal solution of the location routing integrated optimization problem.

This study finds that downstream enterprises' subjective risk attitude has an impact on ordering decisions. The loss aversion coefficient has different impacts on order decision making and maximum expected utility in different periods. In the profit interval, the downstream enterprises increase their expected utility by making active order decisions for risk preference, whereas in the loss interval, the downstream enterprises decrease their expected utility by making conservative order decisions for risk aversion.

Based on the case study of the last mile distribution in Shenyang, China, it is found that the manufacturer is willing to pay the relationship cost higher than $5 \%$ of the minimum assembly and establish a long-term cooperative contract relationship with the downstream enterprises. Manufacturers are no longer simply pursuing the lowest total cost of their own in multicycle cooperation, and they are now willing to pay higher relational costs and gain more longterm profits with partners.

This study found that the expected utility of a multicategory strategy for downstream enterprises is significantly higher than that of a single-product strategy. Multicategory strategies effectively disperse risks and promote utility maximization, and they help participants respond quickly to the supply chain disruption-resistant system and effectively respond to increasingly frequent major public emergencies.
1.5. Structure. This study is organized as follows: Section 2 describes the location routing inventory problem in the context of disruption and provides the necessary definition of the cost-utility uncertainty optimization model for order disruption risk and downstream enterprises' subjective risk preference. Section 3 describes a biobjective linear integerprogramming model with the objective function of minimizing the total cost of the manufacturer and maximizing the subjective utility of the downstream enterprises. Section 4 introduces the implementation of the $\varepsilon$-constraint and compromise algorithm. Section 5 presents an example verification and comparative analysis. Based on randomly generated examples, a series of experiments and comparisons are completed to verify the effectiveness and efficiency of the $\varepsilon$-constraint and compromise algorithm. Section 6 gives the conclusions.

\section{Problem Description and Hypothesis}

This article considers the subjective utility of the downstream firm's risk preference for order delivery disruption to solve the problem that a manufacturer allocates multiple product orders to multiple downstream firms through multiple warehouses.

First, multiproduct order allocation between the manufacturer and downstream firm is completed through multiple cycles, and the downstream firm's demand is uncertain. When the risk of order delivery disruption is uncertain, downstream enterprises pay more attention to increases and decreases of order delivery disruption than in the previous cycle. The downstream firm's utility is based on the order delivery disruption compared with the previous cycle.

Second, vehicles pick up from the warehouse and deliver the orders assigned by the manufacturer to the downstream firms. Each warehouse can store different kinds of goods. Each pickup and delivery vehicle can include a variety of products. After the pickup and delivery service is completed, each vehicle returns to the same open warehouse. Each downstream firm is served only once and only by one vehicle. The goal is to minimize costs, including fixed warehouse costs, fixed vehicle costs, and transportation costs. This study focuses on analysing the impact of order delivery disruption on downstream firm subjective utility considering downstream firm preference. The impact of various parameters on downstream firms' subjective utility should be analysed. To facilitate the analysis of the above problems, the disruption problem of the supply networks will be simplified accordingly. Figure 1 presents a schematic diagram of the supply network structure proposed in this study.

The mathematical model is built on the following practical assumptions. The manufacturer cooperates with $j(j=1,2, \ldots, n)$ downstream firms for $T(T \geq 2)$ cycles. Downstream firms obtain products in the $T(T \geq 2)$ cycle, but not the first cycle, and all products in the whole cooperation cycle. In each cycle, downstream firms $j(j=1, \ldots, n)$ obtain different kinds of products $m(m=1, \ldots, n)$ from the manufacturer. The order delivery disruption of each product 


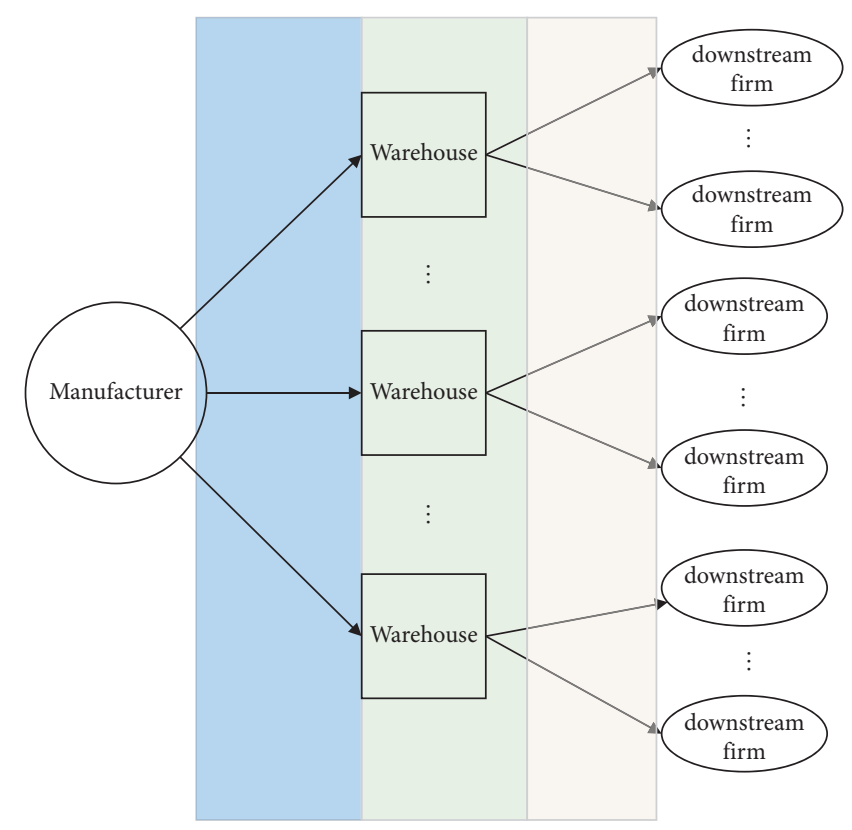

Figure 1: Dynamic structure of the downstream discrete network.

is controlled by the manufacturer $\sum_{j=1}^{n} \zeta_{j m}^{-} \leq R_{j} \leq \sum_{j=1}^{n} \zeta_{j m}^{+}$, $(m=1, \ldots, n)$.

Suppose that each downstream firm's demand for mproducts is independent of each other, and each downstream firm is not related. Downstream firm demand is uncertain and can be described as an order delivery disruption $R_{m} \sim N\left(\mu_{m}, \sigma^{2}\right)$. The product quality allocated by the manufacturer is the same. Product quality will not affect the downstream firm's subjective utility.

The utility of downstream firms for different product combinations is determined according to the previous multicycle cooperation experience. The satisfaction $\gamma_{j m}^{1}$ of downstream firm $j$ in the first period to obtain product $m$ is the same as the subjective utility $v_{1}\left(x_{j m}^{1}\right)$ in the first period.

\section{Subjective Utility of Risk Preference considering Downstream Firms with Order Delivery Disruption}

This section sets the symbolic definition of risk preference considering downstream firms with order delivery disruption according to the problem description and model assumptions. The subjective utility and utility measurement of risk preference considering downstream firms with order delivery disruption. The related parameters of the discrete networks are described in Tables 1-4.

3.1. Downstream Firm Subjective Utility in the First Cycle. In the first cycle, the manufacturer allocates orders to multiple downstream firms. Because there is no reference to the order delivery disruption in the early stage, the downstream firm's risk preference satisfaction of order delivery disruption is taken as the subjective utility function of downstream firms in the first cycle. When the downstream firm obtains the risk $x_{j m}^{1}>\zeta_{j m}^{+}$of order delivery disruption in the first period, the downstream firm's satisfaction in the first period is the ratio of the risk upper limit of product order delivery disruption expected to be obtained to the order delivery disruption brought. When the order delivery disruption is the upper limit of the risk of order delivery disruption, the downstream firm satisfaction is 1 . Therefore, when $\zeta_{j m}^{-}<x_{j m}^{1}<\zeta_{j m}^{+}$, downstream firm satisfaction in the first period is related to initial satisfaction. When $0<x_{j m}^{1}<\zeta_{j m}^{-}$, downstream firm satisfaction is the ratio of the actual risk of order delivery disruption to the lower limit risk of order delivery disruption. In summary, the satisfaction $\gamma_{j m}^{1}$ of the $j$ downstream firm with product $m$ is

$$
\gamma_{j m}^{1}= \begin{cases}\frac{\zeta_{j m}^{+}}{x_{j m}^{1},} & x_{j m}^{1}>\zeta_{j m}^{+}, \\ \gamma_{j m}^{0}+\frac{x_{j m}^{1}-\zeta_{j m}^{-}}{\zeta_{j m}^{+}-\zeta_{j m}^{-}} *\left(1-\gamma_{j m}^{0}\right), & \zeta_{j m}^{-}<x_{j m}^{1}<\zeta_{j m}^{+}, \\ \frac{x_{j m}^{1}}{\zeta_{j m}^{-}} * \gamma_{j m}^{0}, & 0<x_{j m}^{1}<\zeta_{j m}^{-},\end{cases}
$$

where $\gamma_{j m}^{0}$ indicates the initial satisfaction of the downstream firm with the manufacturer when downstream firm $j$ obtains the risk of the disruption of delivery of the $m$ product order from the manufacturer equal to the risk $\zeta_{j m}^{-}$of the leastorder delivery disruption.

Based on the previous assumptions, $j$ downstream firms in phase 1 are satisfied with the risk of obtaining an order delivery disruption for product $m$ as the subjective utility $v_{1}\left(x_{j m}^{1}\right)$ in phase 1 
TABLE 1: Set description of discrete supply networks.

$T=\left\{1,2, \ldots, T^{\max }\right\}$ is the period of cycle, period $t \in T$

$J=\left\{1,2, \ldots, J^{\max }\right\}$ represents the number of downstream firms, downstream firm $j \in J$

$M=\left\{1,2, \ldots, M^{\max }\right\}$ refers to the risk of delivery disruption of an order of a certain product type, $m \in M$ for product

$K=\left\{1,2, \ldots, K^{\max }\right\}$ is the warehouse set, warehouse $k \in K$

$V_{O}=\left\{v_{o 1}, v_{o 2}, \ldots, v_{o}^{\max }\right\}$ represents the warehouse node, warehouse node $v_{o} \in V_{O}$

$V_{C}=\left\{v_{c 1}, v_{c 2}, \ldots, v_{c}^{\max }\right\}$ is the client node, client node $v_{c} \in V_{C}$

$H=\left\{1,2, \ldots, H^{\max }\right\}$ represents the vehicle set, vehicle $h \in H$

$E=\left\{e^{1}, e^{2}, \ldots, e^{\max }\right\}$ is the set of $\operatorname{arcs}, \operatorname{arc} e \in E$

$f_{i j}=$ total load of the vehicle running on the arc $(i, j) \in E$, which is the set of variables $u_{i j}^{h}$ with respect to $H$

TABLE 2: Variable parameter description of discrete supply networks.

$\alpha$ is the decreasing rate of downstream firm sensitivity in the revenue range

$\beta$ is the decreasing rate of downstream firm sensitivity in the loss range

$\lambda$ is the downstream firm's evasion coefficient in case of loss

$\tau_{j m}$ is the preference degree of $j$ downstream firm for product $m$

$w_{j 1}$ is the subjective utility of a single product

$w_{j 2}$ is the subjective utility of downstream firms' multiproducts

$c_{i j}$ is the nonnegative distance of each arc

$D^{k}$ is the storage capacity of the warehouse

$g_{k}$ is the fixed open cost of warehouse

$Q_{h}$ is the vehicle capacity

$f_{h}$ is the fixed vehicle scheduling cost

$u_{i j}^{h}$ is the load of access node $i$ and node $j$

$R_{j m}$ is the risk of order delivery disruption when the manufacturer can provide the $m \in M$ product to the $j$ downstream firm in the $t$ period

$\zeta_{j m}^{-}$is the lower limit of the risk interval value of the delivery disruption of the $m$ product order that the $j$ downstream firm wants to obtain

$\zeta_{j m}^{+}$is the upper limit of the risk interval value of the delivery disruption of the $m$ product order that the $j$ downstream firm wants to obtain

$q_{j}$ means that the $j$ downstream firm has a clear demand

$\gamma_{j m}^{0}$ is the initial satisfaction of downstream firm $j$ to the risk of delivery disruption of the second product order

$\gamma_{j m}^{1}$ represents the satisfaction of $j$ downstream firm to the risk of delivery disruption of $m$ product order in the first cycle

$v_{t}\left(x_{j m}^{t}\right)$ refers to the subjective utility of the $j$ downstream firm that obtains product $m$

TABLE 3: Decision variables description of discrete supply networks.

$x_{j m}^{t}$ represents the risk of disruption of delivery of the $m$ product order assigned by the manufacturer of the $t$ cycle to the $j$ downstream firm

$x_{i j}^{h}$ means that vehicle $h$ is driving from node $i$ to node $j$, which is equal to 1 , with other cases equal to 0

$y_{k}$ indicates whether the warehouse is open, with $y_{k}=1$ indicating that the warehouse is open and 0 indicating that it is not open

$z_{j k}$ indicates whether the downstream firm $j$ is assigned to the warehouse $k$. If the downstream firm $j$ is assigned to the warehouse $k$,

$z_{j k}=1$, otherwise, it is 0

TABle 4: Objective functions description of discrete supply networks.

$Z_{1}$ is the objective function 1

$Z_{2}$ is the objective function 2

$Z_{2}^{*}$ is the optimal value of objective function 2

$$
v_{1}\left(x_{j m}^{1}\right)=\gamma_{j m}^{1}
$$

3.2. Downstream Firm Subjective Utility of the $t$ Cycle. The subjective utility function in the second cycle of downstream enterprises is related not only to the subjective utility in the first cycle but also to the order delivery disruption in the second cycle. If the risk of order delivery disruption in the next cycle is higher than in the previous cycle, the downstream firm's subjective utility will increase.
If the risk of order delivery disruption in the next cycle is lower than that in the previous cycle, the subjective utility of the downstream firm will decrease. Therefore, the subjective utility function of the downstream firm $j$ in the $t(t \geq 1)$ cycle for obtaining product $m$ is

$$
v_{t}\left(x_{j m}^{t}\right)= \begin{cases}\left(\frac{x_{j m}^{t}-x_{j m}^{t-1}}{R_{j m}}\right)^{\alpha}, & x_{j m}^{t} \geq x_{j m}^{t-1}, \\ -\lambda\left(\frac{x_{j m}^{t-1}-x_{j m}^{t}}{R_{j m}}\right)^{\beta}, & x_{j m}^{t}<x_{j m}^{t-1} .\end{cases}
$$

In equation (3), the subjective utility function is the different function of the independent variable $x_{j m}^{t}$, which is the reference value of the subjective utility of the previous period. Let $t=2$ and introduce parallel vertical (1) and formula (2) in equation (3) to obtain the subjective utility 
function of the downstream firm $j$ for product $m$ in the second cycle

$$
v_{2}\left(x_{j m}^{2}\right)=\gamma_{j m}^{1}+\frac{x_{j m}^{2}-x_{j m}^{1}}{R_{j m}} .
$$

The subjective utility function also needs to consider that in the $t(t \geq 2)$ cycle, the subjective utility of downstream firms in this cycle is related not only to the change of the order delivery disruption in the $(t-1)$ cycle but also to the maximum and minimum risk of the order delivery disruption in the $t(t \geq 2)$ cycle.

When $x_{j m}^{t} \geq \zeta_{j m}^{+}$, the risk of the downstream firm's order delivery disruption has exceeded the risk of the downstream firm's desire to obtain the greatest product order delivery disruption. At this time, a lower risk of order delivery disruption corresponds to a greater obtained utility. That is, the subjective utility of the downstream firm should take $\zeta_{j m}^{+}$ as the reference point at this time, not only the $t-1$ cycle, and risk $x_{j m}^{t-1}$ is the reference point.

$$
v_{t}\left(x_{j m}^{t}\right)=1-\lambda\left(\frac{x_{j m}^{t}-\zeta_{j m}^{+}}{R_{j m}}\right)^{\beta} .
$$

In the case of $0 \leq x_{j m}^{t} \leq \zeta_{j m}^{-}$, the risk of order delivery disruption faced by downstream firms is less than the minimum expected risk. In this case, the downstream firm wishes to obtain as many risks of order delivery disruption as possible, so the downstream firm's subjective utility at this time should take $\zeta_{j m}^{-}$as the reference point:

$$
v_{t}\left(x_{j m}^{t}\right)=\lambda\left(\frac{\zeta_{j m}^{-}}{R_{j m}}\right)^{\beta}-\lambda\left(\frac{\zeta_{j m}^{-}-x_{j m}^{t}}{R_{j m}}\right)^{\beta} .
$$

When $0 \leq x_{j m}^{t} \leq \zeta_{j m}^{-}$, take $\zeta_{j m}^{-}$as the reference point to construct the subjective utility function. When the downstream firm's risk of obtaining product order delivery disruption is within the risk range of the downstream firm's desire to obtain product order delivery disruption, that is, $\zeta_{j m}^{-} \leq x_{j m}^{t} \leq \zeta_{j m}^{+}$, then the risk is divided into $x_{j m}^{t}<x_{j m}^{t-1}$ and $x_{j m}^{t} \geq x_{j m}^{t-1}$. When $x_{j m}^{t}<x_{j m}^{t-1}$, the subjective utility of the downstream firm should be chosen to be larger between $\lambda\left(\zeta_{j m}^{-} / R_{j m}\right)$ and $-\lambda\left(x_{j m}^{t-1}-x_{j m}^{t} / R_{j m}\right)^{\beta}$. When the subjective utility of $x_{j m}^{t} \geq x_{j m}^{t-1}$ downstream firms should choose a smaller utility between 1 and $\left(x_{j m}^{t}-x_{j m}^{t-1} / R_{j m}\right)^{\alpha}$, then

$$
\begin{aligned}
& v_{t}\left(x_{j m}^{t}\right)=\max \left[\lambda\left(\frac{\zeta_{j m}^{-}}{R_{j m}}\right)^{\beta},-\lambda\left(\frac{x_{j m}^{t-1}-x_{j m}^{t}}{R_{j m}}\right)^{\beta}\right], \\
& v_{t}\left(x_{j m}^{t}\right)=\min \left[1,\left(\frac{x_{j m}^{t}-x_{j m}^{t-1}}{R_{j m}}\right)^{\alpha}\right] .
\end{aligned}
$$

When $x_{j m}^{t}=\zeta_{j m}^{-}$, the downstream firm's subjective utility is determined by the downstream firm's desire to obtain the lowest order delivery disruption risk, the $j$ product order delivery disruption risk, the sensitivity reduction speed, and the loss avoidance coefficient when the downstream firm obtains the product order delivery disruption risk reduction, namely, $\lambda\left(\zeta_{j m}^{-} / R_{j m}\right)^{\beta}$. When $x_{j m}^{t}=\zeta_{j m}^{+}$, the downstream firm's subjective utility reaches the highest value of 1 . In summary, the subjective utility function of downstream firm $j$ in the $t(t \geq 1)$ cycle for obtaining product $m$ is as follows:

$$
v_{t}\left(x_{j m}^{t}\right)= \begin{cases}1-\lambda\left(\frac{x_{j m}^{t}-\zeta_{j m}^{+}}{R_{j m}}\right)^{\beta}, & x_{j m}^{t} \geq \zeta_{j m}^{+}, \\ \min \left[1,\left(\frac{x_{j m}^{t}-x_{j m}^{t-1}}{R_{j m}}\right)^{\alpha}\right], & x_{j m}^{t} \geq x_{j m}^{t-1}, \\ \max \left[\lambda\left(\frac{\zeta_{j m}^{-}}{R_{j m}}\right)^{\beta},-\lambda\left(\frac{x_{j m}^{t-1}-x_{j m}^{t}}{R_{j m}}\right)^{\beta}\right], & x_{j m}^{t}<x_{j m}^{t-1}, \\ \lambda\left(\frac{\zeta_{j m}^{-}}{R_{j m}}\right)-\lambda\left(\frac{\zeta_{j m}^{-}-x_{j m}^{t}}{R_{j m}}\right)^{\beta}, & 0 \leq x_{j m}^{t} \leq \zeta_{j m}^{-} .\end{cases}
$$

3.3. Downstream Firm Subjective Utility Function. Downstream firms have different preferences for the subjective utility of the order delivery disruption risk for different products and product types; therefore, the subjective utility of downstream firms should be divided into the subjective utility of risk of disruption of single product order delivery and the subjective utility of risk of disruption of multiproduct order delivery.

Downstream firms have different preferences for the subjective utility of different products and different types of order delivery disruption risk. Therefore, the subjective utility of downstream firms is divided into the subjective 
utility of single-product order delivery disruption risk and multiple-product order delivery disruption risk. Suppose that the subjective utility weight of a single product for downstream firm $j$ is $w_{j 1}$, the subjective utility weight of multiple products is $w_{j 2}$, and $w_{j 1}+w_{j 2}=1$. The specific values of $w_{j 1}$ and $w_{j 2}$ can be obtained by long-term followup investigation of downstream firms.

Downstream firms' subjective utility consists of two parts: single-product subjective utility $w_{j 1}$ and multiproduct subjective utility $\left(w_{j 2} / n\right)$. First, for a single product, different downstream firms have different subjective utility preferences for different products. Let the $j$ downstream firm's subjective preference for product $m$ be $\tau_{j m}$, and let $\sum_{m=1}^{n} \tau_{j m}=1$; then, let the downstream firm $j$ be in the $t$ cycle. The subjective utility of a single product is $w_{j 1} \sum_{m=1}^{n} \tau_{j m} v_{t}\left(x_{j m}^{t}\right)$. Second, for multiple products, the subjective utility function of multiple products refers to the degree of downstream firm preference for the risk of order delivery disruption of product categories. In real life, downstream firms are often more willing to provide a wide range of products for sale, thus making the product portfolio richer by the inclusion of more alternatives for one product and more choices for downstream firms and downstream firms. In general, a larger average value of the risk of disruption of the product distribution order delivery obtained by the downstream firm corresponds to a greater number of product types and product portfolios that will be available to the downstream firm and a higher subjective utility function of the multiple products. The subjective utility function of the downstream firm $j$ for multiple products in cycle $t$ is $\left(w_{j 2} / n\right) \sum_{m=1}^{n} v_{t}\left(x_{j m}^{t}\right)$.

Therefore, the overall subjective utility function of the risk preference of the downstream firm order delivery disruption in cycle $t$ is given as

$$
v_{t}(j)=w_{j 1} \sum_{m=1}^{n} \tau_{j m} v_{t}\left(x_{j m}^{t}\right)+\frac{w_{j 2}}{n} \sum_{m=1}^{n} v_{t}\left(x_{j m}^{t}\right) .
$$

\section{Compromise Algorithm Design}

For the uncertain optimization model considering the risk preference of a downstream firm's order delivery disruption, we constructed a biobjective mixed integer-programming model to maximize the downstream firm's subjective utility and minimize the manufacturer's cost, which transformed the model to some extent.

4.1. Biobjective Optimization Modelling. The specific uncertain optimization biobjective model is described considering the risk preference of the downstream firm's order delivery disruption. The downstream firm's subjective utility $Z_{1}$ is maximized, and the manufacturer's distribution cost $Z_{2}$ is minimized. The manufacturer's distribution cost includes the fixed cost of the warehouse, the fixed cost of the vehicle, and the variable cost of transportation.

$$
\begin{aligned}
& Z_{1}=\max \frac{1}{T} \sum_{j=1}^{n} \sum_{m=1}^{n} \sum_{t=1}^{T} v_{t}(j), \\
& Z_{2}=\min \left(\sum_{k \in V_{O}} g_{k} y_{k}+\sum_{h \in H} \sum_{k \in V_{O}} \sum_{j \in V_{C}} f_{h} x_{i j}^{h}\right. \\
& \left.+\sum_{h \in H} \sum_{(i, j) \in E} c_{i j} x_{i j}^{h}\right), \\
& \sum_{h \in H} \sum_{i \in V_{O}} x_{i j}^{h}=1, j \in V_{C}, \\
& \sum_{h \in H} \sum_{i \in V_{O}} x_{i j}^{h}=\sum_{h \in H} \sum_{i \in V_{O}} x_{j i}^{h}, j \in V_{C}, \\
& \sum_{h \in H} \sum_{i \in V_{O}} u_{i j}^{h}-\sum_{h \in H} \sum_{i \in V_{O}} u_{j i}^{h}=q_{j}, j \in V_{C}, \\
& \sum_{i j}^{h} \geq q_{i} x_{i j}^{h}, i \in V_{O}, j \in V_{C}, h \in D^{k} y_{k}, k \in K_{i j}, \\
& \sum_{h \in H}^{h} \leq Q_{i \in V_{O}} x_{i j}^{h}, i \in V_{O}, j \in V_{C}, i \neq j, h \in H, \\
& \sum_{h \in H} \sum_{i \in V_{O}} u_{k i}^{h}=\sum_{j \in V_{C}} Z_{j k} q_{j}, k \in K, \\
& \left.\sum_{h}-q_{j}\right) x_{i j}^{h}, i \in V_{O}, j \in V_{C}, h \in H,
\end{aligned}
$$

$\sum_{h^{\prime} \in H, h^{\prime} \neq h} \sum_{i^{\prime} \in V_{O}, i^{\prime} \neq i} x_{i i}^{h^{\prime}}+x_{i j}^{h} \leq 1, i \in V_{O}, j \in V_{C}, i \neq j, h \in H$,

$\sum_{h \in H} x_{j k}^{h} \leq Z_{j k}, k \in K, h \in H$

$$
\sum_{h \in H} x_{k j}^{h} \leq Z_{j k}, k \in K, j \in V_{C}
$$

$\sum_{h \in H} x_{i j}^{h}+Z_{j k}+\sum_{k^{\prime} \in K, k^{\prime} \neq k} Z_{i k^{\prime}} \leq 2, k \in K,(i, j) \in E$,

$u_{i j}^{h} \geq 0,(i, j) \in E, h \in H$

$x_{i j}^{h} \in\{0,1\},(i, j) \in E, h \in H$, 


$$
\begin{gathered}
Z_{j k} \in\{0,1\}, k \in K, j \in V_{C}, \\
y_{k} \in\{0,1\}, k \in K, \\
\sum_{m=1}^{n} x_{j m}^{t}=\mu_{m}+\phi^{-1}(\alpha) \sigma_{m}, \\
x_{j m}^{t} \geq 0,
\end{gathered}
$$$$
\alpha \in(0,1), \beta \in(0,1), \lambda>1 \text {. }
$$

Constraints (13) and (14) are degree restrictions. In particular, constraint (13) ensures that each client point is visited only once. Constraint (14) ensures that the arcs lead to equivalent points in and out. Constraint (15) means that each downstream firm of order is fully delivered. Constraint (16) means that the total load of any arc cannot exceed the vehicle's capacity. Constraint (17) ensures that the total load of each warehouse is equal to the total demand of its downstream firms. Note that constraint (17) strengthens the constraint condition, but it is unnecessary and will be simplified in the subsequent solution process. Constraint (18) specifies that the vehicle load returned to each warehouse must be equal to 0. Constraints (19) and (20) are boundary constraints of load variables. Constraint (21) ensures that the total demand and supply of the warehouse cannot exceed its capacity. Constraints (22) and (23) ensure that each downstream firm is assigned to only one warehouse and one vehicle type, respectively. Constraints (24)-(26) prohibit unreasonable routes. Routes should start and end in different warehouses. Constraints (27)-(30) define the range of decision variables. The effectiveness of constraints (24)-(26) prevents unreasonable routes from one warehouse to another.

4.2. Biobjective Compromise Algorithm. Considering the risk preference of downstream firms of order delivery disruption, the abovementioned uncertain optimization model is a biobjective mixed-integer optimization model. Two objectives of the model are the downstream firm's subjective utility and the manufacturer's delivery cost. Two objectives of the model have certain dependencies but are not commensurable in units. Therefore, the idea of solving the biobjective linear hybrid optimization model is to transform the biobjective into a single-objective optimization model.

Step 1: Simplify the manufacturer's distribution model: the distribution models (12)-(30) are adequate for the manufacturer's distribution problem.

Step 1.1: Let (12) be E1, and then, use some variables or decompose some constraints to simplify the model. The scale of the formula is reduced by variables, and the loose boundary is tightened by decomposing some constraints.

Step 1.2: Then, $f_{i j}$ is a collection of variables $u_{i j}^{h}$, and the following is performed:

$$
\begin{gathered}
\sum_{i \in V_{O}} f_{i j}-\sum_{i \in V_{O}} f_{j i}=q_{j}, j \in V_{C}, \\
f_{i j} \leq Q_{h} x_{i j}^{h}, i \in V_{O}, j \in V_{C}, i \neq j, \\
\sum_{i \in V_{O}} f_{k i}=\sum_{i \in V_{O}} Z_{i k} q_{i}, k \in K, \\
\sum_{i \in V_{O}} f_{i k}=0, k \in K, \\
\sum_{h \in H}\left(Q_{h}-q_{j}\right) x_{i j}^{h}, i \in V_{O}, j \in V_{C}, \\
f_{i j} \geq q_{i} \sum_{h \in H} x_{i j}^{h}, i \in V_{O}, j \in V_{C} .
\end{gathered}
$$

Let $(f, x)$ be the solution satisfying (34)-(39) and $(u, x)$ be the solution satisfying constraints (13) and (15)-(20), where $f, u$, and $x$ are $f_{i, j}$ and $x_{i j}^{h}$, respectively. Then, for every feasible solution $(u, x)$, there is a feasible solution $(f, x)$ and vice versa. Based on (13), $x_{i j}^{h *}=1, h \in H$, and based on (19), $u_{i j}^{h *} \geq 0$, which means that $x_{i j}^{h}=u_{i j}^{h}=0, h \in H$.

Step 1.3: Using these parameters, the way to contact $f_{i j}$ and the original variables is as follows:

$$
f_{i j}=\sum_{h \in H} u_{i j}^{h}, i \in V_{O}, j \in V_{C} .
$$

Step 1.4: Using formula (40), constraints (34)-(39) can replace constraints $(15)-(20)$.

$$
\sum_{i \in V_{O}} x_{i j}^{h}=\sum_{i \in V_{O}} x_{j i}^{h}, j \in V_{C}, h \in H .
$$

Step 1.5: Constraint (41) is equivalent to constraints (14) and (23). Given that $i, j$, and $k$ have the same route and assuming that $h^{1}, h^{2} \in H, x_{i j}^{h 1}=1$, and $x_{i k}^{h 2}=1$, the constraint is valid for (14). However, constraints (13) and (14) only allow the same vehicle to be used on the route, that is, $x_{i j}^{h 1}=1$ and $x_{i k}^{h 2}=1$. We can now obtain constraint (41) from (14). The use of (41) makes (23) redundant. Constraint (41) guarantees $x_{i j}^{h 1}=1$ and $x_{i k}^{h 2}=1$ without using (23). In this case, the manufacturer's cost function in the distribution process includes the objective function $E 1$, and the constraints are (13), (14), (21)-(30), and (34)-(39). Next, the two-objective optimization model considering the risk preference of downstream firms of order delivery disruption is simplified.

Step 2: Implement the $\varepsilon$-constraint method.

Step 2.1: Suppose that the biobjective programming of the discrete network problem is as follows:

$$
\begin{array}{ll}
\max & \left(f_{1}(x), f_{2}(x), \ldots \ldots, f_{p}(x)\right), \\
\text { s.t. } & X \in S .
\end{array}
$$


$X$ is the vector of decision variables, $f_{1}(x), f_{2}(x), \ldots, f_{p}(x)$ is the objective function, and $S$ is the feasible region.

Step 2.2: In step 2 of the $\varepsilon$ constraint method, an objective function is optimized, and the rest of the objective functions are taken as constraints.

$$
\begin{array}{ll}
\max & f_{1}(x), \\
\text { s.t. } & f_{2}(x) \geq e_{2}, \\
& f_{3}(x) \geq e_{3}, \\
& f_{p}(x) \geq e_{p}, \\
X & \in S .
\end{array}
$$

The feasible solution of the optimization problem is obtained by constraining the parameter $\left(e_{i}\right)$ of the objective function. Compared with the weighted method, the $\varepsilon$ constraint method has the following advantages. For linear problems, the weighted method is applied to the original feasible region and obtains a point solution (the solution of the extreme point); therefore, it will generate a unique extreme point solution. In contrast, the $\varepsilon$ constraint method can change the original feasible region and generate nonextreme solutions. The result is that the weighted method may spend a considerable amount of redundant time running multiple weight combinations but obtain the same extreme point solution. Moreover, the $\varepsilon$ constraint method can generate different feasible solutions to obtain a more abundant set of feasible solutions. The weighted method cannot solve the biobjective mixed-integer programming problem. However, the $\varepsilon$ constraint method can solve the biobjective mixed-integer programming problem effectively. Therefore, it is not necessary to obtain the uniform objective function before the weighted common degree method. An additional advantage of the $\varepsilon$ constraint method is that it can control the risk of order delivery disruption to produce a feasible solution by adjusting the risk of order delivery disruption in each objective function.

Step 3: The biobjective is transformed into a single objective.

Step 3.1: The objective function $Z_{1}$ is not considered; rather, only the objective function $Z_{2}$ is considered. The manufacturer is allowed to bear a higher $\delta$ relationship cost than the minimum value $Z_{2}^{*}$ of the objective function $Z_{2}$, and $\delta$ is the cost coefficient of the objective function. In this case, $Z_{2}$ can be transformed as follows:

$$
Z_{2} \leq(1+\delta) Z_{2}^{*}
$$

where $Z_{2}^{*}$ is the minimum value of the objective function $Z_{2}$, and $\delta$ is a small positive number. Transforming $Z_{2}$ into $Z_{2} \leq(1+\delta) Z_{2}^{*}$ means that manufacturers do not have to support the lowest cost but can support values slightly higher than the lowest cost to maintain a good relationship with downstream firms in the multicycle cooperation between manufacturers and downstream firms. More profits can be obtained through multicycle cooperation. For example, $\delta=0.05$, which means that manufacturers are willing to support the relationship cost $5 \%$ higher than the lowest cost to maintain a good relationship with downstream firms.

Step 3.2: The uncertain optimization biobjective mixed-integer model considering the risk preference of the downstream firm's order delivery disruption can be transformed as follows:

$$
\begin{aligned}
& \qquad Z_{1}=\max \frac{1}{T} \sum_{j=1}^{n} \sum_{m=1}^{n} \sum_{t=1}^{T}\left[w_{j 1} \sum_{m=1}^{n} \tau_{j m} v_{t}\left(x_{j m}^{t}\right)+\frac{w_{j 2}}{n} \sum_{m=1}^{n} v_{t}\left(x_{j m}^{t}\right)\right], \\
& \text { s.t. } \quad Z_{2} \leq(1+\delta) Z_{2}^{*}, \\
& \sum_{m=1}^{n} x_{j m}^{t}=\mu_{m}+\phi^{-1}(\alpha) \sigma_{m} \\
& \quad x_{j m}^{t} \geq 0, \\
& \alpha \in(0,1), \beta \in(0,1), \lambda>1 .
\end{aligned}
$$


Step 4: After the biobjective compromise, the search operator searches in the solution space and obtains $x_{j m}^{t}$, $x_{i j}^{h}, y_{k}$, and $z_{j k}$ after the compromise steps.

\section{Numerical Experiment}

5.1. Experimental Data and Parameter Setting. In the longterm multicycle cooperation between the manufacturer and the downstream firm, the manufacturer pays more attention to the subjective utility of the downstream firm. The allocated cost is allowed to exceed a certain price coefficient. Annual demand is shown in Figure 2 for several large cities.

For this demand, the manufacturer is assumed to provide products to downstream firms for five kinds of products $A_{1}, A_{2}, A_{3}, A_{4}$, and $A_{5}$. The parameters, including the cost coefficients, are $c_{j 1}, c_{j 2}$, and $c_{j 3}$. Ideal intervals are $\left[\zeta_{j 1}^{-}, \zeta_{j 1}^{+}\right]$, $\left[\zeta_{j 2}^{-}, \zeta_{j 2}^{+}\right]$, and $\left[\zeta_{j 3}^{-}, \zeta_{j 3}^{+}\right]$. Product utilities are $\tau_{j 1}, \tau_{j 2}$, and $\tau_{j 3}$. The utility weights are $w_{j 1}$ and $w_{j 2}$, and the initial satisfactions are $\gamma_{j 1}^{0}, \gamma_{j 2}^{0}$, and $\gamma_{j 3}^{0}$. The parameters of downstream firms are shown in Table 5.

5.2. Sensitivity Analysis. It has been assumed that distance, travel time, and travel cost can be used interchangeably in the distribution process. For a simple network, the BPR (bureau of public roads) function $t_{a}\left(x_{a}\right)=t_{a}^{0}\left[1+0.15\left(x_{a} / c_{a}\right)^{4}\right]$ is used, where $x_{a}$ is the flow of side $a, t_{a}^{0}$ is the normal travel time of side $a$, and $c_{a}$ is the maximum trafficable flow of side $a$. Assuming that the distribution of travel time is independent and $\sigma_{a}^{2}=\varphi_{a} t_{a}$, $\varphi_{a}\left(\varphi_{a}>0\right)$ is the variance of travel time, the specific parameter values are shown in Table 6 .

When $\alpha=0.79$ and $\beta=0.62$ are verified, the influence of $\lambda$ changes in the first three cycles on downstream firms' subjective utility is shown in Table 7.

5.3. Comparative Analysis. The nondominated sorting genetic algorithm with an elite strategy is a multiobjective evolutionary algorithm proposed by Deb et al. [23], and it has a fast and accurate search performance. The genetic mechanism-based NSGA-II algorithm of Ghannadpour and Zarrabi [24] is easy to adapt to the specific requirements of various objective functions. It can quickly find good solutions to high-dimensional problems; therefore, NSGA-II is often used to solve complex multiobject vehicle routing problems, and it has universal applicability. Therefore, the NSGA-II algorithm is selected and compared with the $\varepsilon$-constraint method.

In this section, the mechanism of the NSGA-II algorithm in the Ghannadpour and Zarrabi studies will be used for comparison. Due to uncertain discrete supply networks in the postepidemic period, the encoding and decoding operations of the NSGA-II algorithm need to be adjusted to include vehicle information. When coding, the complete transportation plan is coded into 1 chromosome with a real number, the real number corresponds to the order number, and the value of 0 is not used for division in the middle.
When the chromosome is decoded, one chromosome is divided into multiple routes according to the feasibility conditions, and different vehicles complete the transportation task. The feasibility condition is the capacity limit of the vehicle; that is, when the load exceeds the maximum capacity, the chromosomes are cut to form a new transportation route, and new vehicles are sent to complete the transportation. Each time a vehicle is dispatched, the model with a larger capacity is preferred. In this way, each route is matched by a vehicle of the corresponding model. If the scheme after matching the current car model with the route is not feasible, a new car model arrangement is randomly generated and matched with the route. The result of running under the same experimental environment is the optimal value of 30 runs. Table 8 compares the optimal solution of the risk threshold cost of the two algorithms. The transportation cost and transportation risk of the optimal solution of the risk threshold cost of the $\varepsilon$-constraint method are lower than those of the NSGA-II algorithm. Therefore, the quality of the Pareto solution set obtained by the $\varepsilon$-constraint method is significantly better than the result of the NSGA-II algorithm.

To further compare the performance of the two algorithms, the solution set coverage and the maximum dispersion are selected to evaluate the experimental results of the eight groups of examples. The solution set coverage rate proposed by Zitzler and Thiele [25] is the most commonly used evaluation method to express the ratio of the solution set $v$ dominated by at least one solution in the solution set $u$. If the index is 1 , all solutions in $v$ are dominated by solutions in $u$. Maximum dispersion is a method proposed by Zitzler et al. [26] to evaluate the spread of the solution set. This index calculates the distance between extreme individuals in the nondominated solution set. In addition, Table 9 compares the running time of the algorithm, and the running time is the average of 30 experiments. The results in Tables 8 and 9 show the following:

First, the results obtained by the $\mathcal{E}$-constraint method perform better in the optimal solution of the risk threshold cost and the solution set coverage index, which shows that under the same risk level, the $\varepsilon$-constraint method can provide enterprises with more considerable cost savings. Therefore, enterprises are more willing to choose low-risk transportation solutions, thereby effectively reducing the transportation risks.

Second, for the maximum dispersion index, in addition to example 1, the boundary solution of the Pareto front surface obtained by the $\mathcal{E}$-constraint method has a larger span and a wider distribution. To a certain extent, the search range of the solution space of the $\varepsilon$-constraint method is wider than that of the NSGA-II algorithm, which reflects the superiority of the $\varepsilon$-constraint method.

Third, the $\varepsilon$-constraint method runs faster. However, under the scale of the current calculation example, the running time of the two algorithms is relatively short. Therefore, this difference will not have a major impact on the performance of the NSGA-II algorithm. 


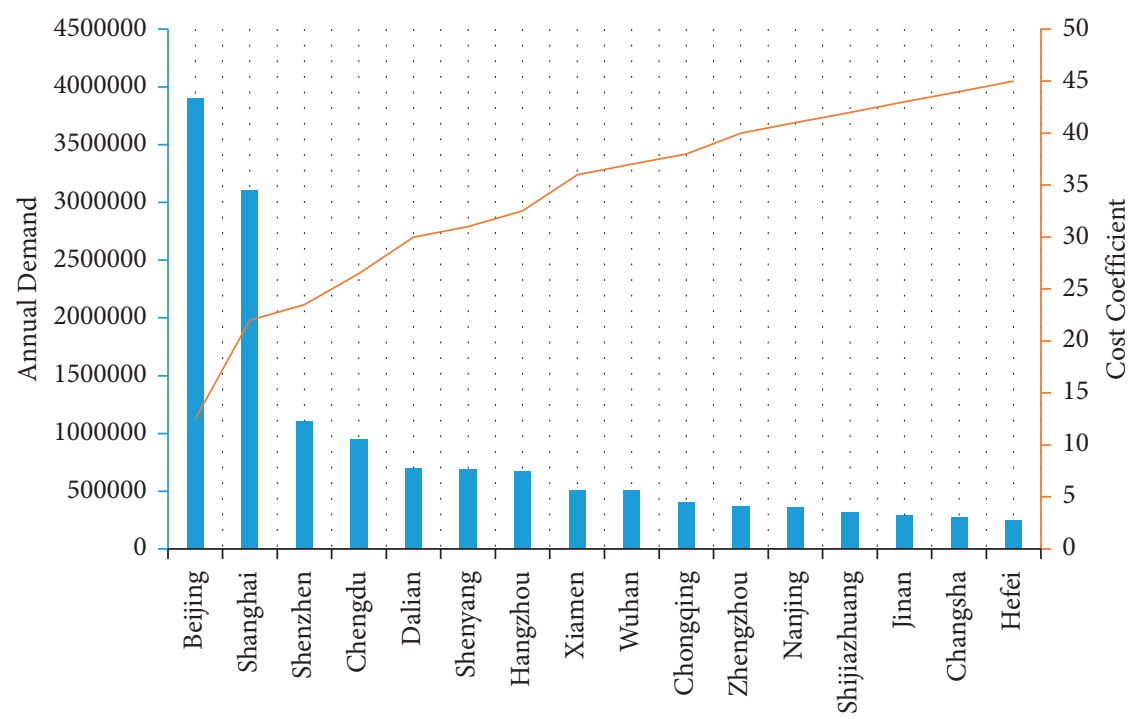

FIgURE 2: Illustration of annual demand in several big cities.

TABle 5: Parameter values.

\begin{tabular}{lccccc}
\hline & $A_{1}$ & $A_{2}$ & $A_{3}$ & $A_{4}$ & $A_{5}$ \\
\hline$c_{j 1}$ & 20 & 21 & 20 & 25 & 36 \\
$c_{j 2}$ & 35 & 33 & 35 & 25 & 33 \\
$c_{j 3}$ & 20 & 22 & 22 & 0.35 & 0.25 \\
$\tau_{j 1}$ & 0.33 & 0.32 & 0.33 & 0.25 & 0.34 \\
$\tau_{j 2}$ & 0.25 & 0.25 & 0.24 & 0.51 & 0.35 \\
$\tau_{j 3}$ & 0.25 & 0.36 & 0.36 & 0.50 & 0.35 \\
$w_{j 1}$ & 0.43 & 0.52 & 0.45 & 0.38 \\
$w_{j 2}$ & 0.61 & 0.53 & 0.35 & 0.45 \\
$\gamma_{j 1}^{0}$ & 0.24 & 0.35 & 0.44 & 0.35 \\
$\gamma_{j 2}^{0}$ & 0.35 & 0.35 & 0.35 & {$[15,45]$} \\
$\gamma_{j 3}^{0}$ & 0.23 & 0.25 & {$[25,40]$} & {$[20,40]$} \\
{$\left[\zeta_{j 1}^{-}, \zeta_{j 1}^{+}\right]$} & {$[60,80]$} & {$[25,40]$} & {$[50,80]$} & 0.47 \\
{$\left[\zeta_{j 2}^{-}, \zeta_{j 2}^{+}\right]$} & {$[10,20]$} & {$[20,35]$} & {$[20,45]$} & {$[15,40]$} \\
{$\left[\zeta_{j 3}^{-}, \zeta_{j 3}^{+}\right]$} & {$[20,40]$} & {$[15,35]$} & & {$[20,60]$} \\
\hline
\end{tabular}

TABLe 6: Parameter values.

\begin{tabular}{lccc}
\hline & $t_{a}^{0}(\min )$ & $c_{a}($ veh/h) & $\varphi_{a}$ \\
\hline 1 & 20 & 4000 & 0.56 \\
2 & 22 & 3000 & 0.65 \\
3 & 22 & 5000 & 0.62 \\
4 & 18 & 5000 & 0.82 \\
5 & 20 & 3000 & 0.64 \\
6 & 20 & 3000 & 0.54 \\
7 & 10 & 4000 & 0.64 \\
8 & 12 & 4000 & 0.62 \\
9 & 14 & 4000 & 0.25 \\
10 & 10 & 3000 & 0.33 \\
11 & 10 & 5000 & 0.72 \\
12 & 10 & 5000 & 0.83 \\
\hline
\end{tabular}


TABLE 7: The impact of $\lambda$ change on downstream firms' subjective utility.

\begin{tabular}{lcccccrr}
\hline$\lambda$ & $t=1$ & $t=2$ & $t=3$ & $\lambda$ & $t=1$ & $t=2$ & 0.83 \\
2.10 & 0.62 & 0.63 & 0.61 & 2.10 & 0.85 & 0.81 \\
2.20 & 0.64 & 0.62 & 0.72 & 2.20 & 0.69 & 0.62 \\
2.30 & 0.61 & 0.60 & 0.69 & 2.30 & 0.69 & 0.68 \\
2.40 & 0.69 & 0.64 & 0.57 & 2.40 & 0.78 & 0.78 \\
2.50 & 0.61 & 0.61 & 0.67 & 2.50 & 0.77 & 0.72 \\
2.60 & 0.61 & 0.65 & 0.67 & 2.60 & 0.64 & 0.73 \\
2.70 & 0.68 & 0.69 & 0.51 & 2.70 & 0.76 & 0.71 \\
2.80 & 0.60 & 0.68 & 0.70 & 2.80 & 0.73 & 0.78 \\
2.90 & 0.66 & 0.61 & 0.69 & 2.90 & 0.75 & 0.77 \\
3.00 & 0.67 & 0.66 & 0.86 & 3.00 & 0.70 & 0.76 \\
\hline
\end{tabular}

TABLE 8: Comparison of optimal $Z_{1}$ and $Z_{2}$ for 8 groups of examples.

\begin{tabular}{lcccr}
\hline & \multicolumn{2}{c}{$\varepsilon$-constraint method } & & \multicolumn{1}{c}{ NSGA-II } \\
NO. & $Z_{1}$ & $Z_{2}$ & $Z_{1}$ & 120.6 \\
\hline 1 & 5639.6 & 100.5 & 3921.3 & 117.8 \\
2 & 4279.4 & 98.2 & 3851.5 & 11.0 \\
3 & 4193.4 & 92.5 & 3774.1 & 108.8 \\
4 & 3903.5 & 90.7 & 3513.2 & 106.9 \\
5 & 3872.1 & 89.1 & 3484.9 & 102.4 \\
6 & 3798.2 & 85.3 & 3418.4 & 1.4 \\
7 & 3590.4 & 83.6 & 3231.4 & 100.3 \\
8 & 3381.2 & 81.5 & 3043.1 & 120.6 \\
\hline
\end{tabular}

TABLE 9: Results of the comparison between the $\varepsilon$-constraint method and NSGA-II indexes.

\begin{tabular}{|c|c|c|c|c|c|}
\hline NO. & $\begin{array}{c}\text { Solution set } \\
\text { coverage }\end{array}$ & $\begin{array}{c}\text { Maximum single-layer } \\
\text { distribution threshold value of } \\
\varepsilon \text {-constraint method }\end{array}$ & $\begin{array}{c}\text { Maximum single-layer } \\
\text { distribution threshold value of } \\
\text { NSGA-II }\end{array}$ & $\begin{array}{l}\text { Running time of } \\
\varepsilon \text {-constraint method per } \\
\text { second }\end{array}$ & $\begin{array}{l}\text { Running time of } \\
\text { NSGA-II per } \\
\text { second }\end{array}$ \\
\hline 1 & 0.86 & 3549.2 & 5732.4 & 12.04 & 13.2 \\
\hline 2 & 1.00 & 25356.7 & 7329.7 & 16.58 & 18.2 \\
\hline 3 & 1.00 & 11291.3 & 6842.3 & 13.07 & 14.4 \\
\hline 4 & 0.85 & 10675.8 & 7789.3 & 15.81 & 17.4 \\
\hline 5 & 1.00 & 27577.2 & 8438.0 & 16.93 & 18.6 \\
\hline 6 & 0.90 & 15765.1 & 12213.5 & 18.45 & 20.3 \\
\hline 7 & 0.95 & 20321.3 & 13217.9 & 18.39 & 20.2 \\
\hline 8 & 1.00 & 21345.1 & 12236.8 & 18.66 & 20.5 \\
\hline
\end{tabular}

\section{Conclusion}

In this work, we studied the uncertain optimization of discrete networks with order delivery disruption and risk preference in the postepidemic era. We suggest that the downstream firm's subjective utility on the risk of order delivery disruption is a reference point and measure the utility of the downstream firm's risk preference on order delivery disruption. Moreover, we constructed the downstream firm's subjective utility function and the biobjective optimization model to maximize the downstream firm's subjective utility and minimize the manufacturer's cost. The influence of each parameter in the downstream firm's subjective utility function on the uncertain optimization is analysed.

The results show that when the subjective utility of downstream firms in the first cycle and the second cycle is the same at $\lambda$, the subjective utility of downstream firms in the second cycle is less than that in the first cycle. Nevertheless, the changing trend is not the same. At the same time, the change of subjective utility of downstream firms in the third cycle and the second cycle is not the same as that in the second cycle and the first cycle, and a rule is not available to follow. The first cycle is the initial cycle. In the model mentioned in this section, the downstream firm's initial satisfaction is determined according to the minimum value of the order interval, which is obtained based on the expected utility theory of the rational person. In the second cycle, the downstream firm's order quantity preference is introduced, and the downstream firm is no longer completely rational. The downstream firm has a risk preference for the income interval and a risk aversion for the loss interval. However, in the profit interval, the increase in subjective utility due to an increase in order quantity is less than the decrease in subjective utility due to a decline in order quantity in the loss interval; therefore, the subjective 
utility of downstream firms in the second cycle will be lower at the same $\lambda$. A change of the third cycle preliminarily indicates that $\lambda$ has little effect on the change of downstream firms' subjective utility.

Future research should focus on extending the length of discrete networks and the segmentation of enterprise risk preference. In addition, designing a discrete network coordination mechanism based on the moral or social responsibility of suppliers is also a topic for future scholars.

\section{Data Availability}

All the data generated or analysed during this study are included in this article. The data used to support the findings of this study have been deposited in the [https://pan.baidu. com/s/1fz4M0Bjc8KJkPFSTgRXe0A (password:pm0t)] repository ([DOI or OTHER PERSISTENT IDENTIFIER]).

\section{Conflicts of Interest}

The authors declare that they have no conflicts of interest.

\section{Acknowledgments}

This work was partly supported by the National Natural Science Foundation of China (Project Nos. 70431003), the Science-Technology Major Project of Liaoning Province China (Project Nos. 2019JH1/10100028), the Talent Project of Revitalizing Liaoning (Project Nos. XLYC1808044), Scientific Research Foundation Program of the Educational Department of Liaoning Province China (Project Nos. JYT2019-02), and Science-Technology Planning Project of Shenfu Reform and Innovation Demonstration Zone of Liaoning Province China (Project Nos. 2020JH19).

\section{References}

[1] X. Gan, S. P. Sethi, and H. Yan, "Coordination of supply chains with risk-averse agents," Production and Operations Management, vol. 13, no. 2, pp. 135-149, 2004.

[2] X. Qi, J. F. Bard, and G. Yu, "Supply chain coordination with demand disruptions," Omega, vol. 32, no. 4, pp. 301-312, 2004.

[3] T. Xiao, G. Yu, Z. Sheng, and Y. Xia, "Coordination of a supply chain with one-manufacturer and two-retailers under demand promotion and disruption management decisions," Annals of Operations Research, vol. 135, no. 1, pp. 87-109, 2005.

[4] H. Fu, Y. Ma, D. Ni, and X. Cai, "Coordinating a decentralized hybrid push-pull assembly system with unreliable supply and uncertain demand," Annals of Operations Research, vol. 257, no. 4, pp. 537-557, 2015.

[5] J. Chen, H. Wang, and R. Y. Zhong, "A supply chain disruption recovery strategy considering product change under COVID-19," Journal of Manufacturing Systems, vol. 60, no. 5, 2021.

[6] T. Sawik, "Joint supplier selection and scheduling of customer orders under disruption risks: single vs. dual sourcing," Omega, vol. 43, no. 3, pp. 83-95, 2014.

[7] L. V. Snyder, Z. Atan, P. Peng, Y. Rong, A. J. Schmitt, and B. Sinsoysal, "OR/MS models for supply chain disruptions: a review," IIE Transactions, vol. 48, no. 2, pp. 89-109, 2015.
[8] J.-S. Song and P. Zipkin, "Inventories with multiple supply sources and networks of queues with overflow bypasses," Management Science, vol. 55, no. 3, pp. 362-372, 2009.

[9] F. Rayat, M. Musavi, and A. Bozorgi-Amiri, "Bi-objective reliable location-inventory-routing problem with partial backordering under disruption risks: a modified AMOSA approach," Applied Soft Computing, vol. 59, no. 10, pp. 622-643, 2017.

[10] B. Tomlin, "On the value of mitigation and contingency strategies for managing supply chain disruption risks," Management Science, vol. 52, no. 5, pp. 639-657, 2006.

[11] X. Gan, S. Sethi, and H. Yan, "Channel coordination with a risk-neutral supplier and a downside-risk-averse retailer," Production and Operations Management, vol. 14, no. 1, pp. 80-89, 2010.

[12] H.-S. Lau and A. H.-L. Lau, "Manufacturer's pricing strategy and return policy for a single-period commodity," European Journal of Operational Research, vol. 116, no. 2, pp. 291-304, 1999.

[13] V. Agrawal and S. Seshadri, "Impact of uncertainty and risk aversion on price and order quantity in the newsvendor problem," Manufacturing \& Service Operations Management, vol. 2, no. 4, pp. 410-423, 2000.

[14] A. A. Tsay, "Risk sensitivity in distribution channel partnerships: implications for manufacturer return policies," Journal of Retailing, vol. 78, no. 2, pp. 147-160, 2002.

[15] A. Xanthopoulos, D. Vlachos, and E. Iakovou, "Optimal newsvendor policies for dual-sourcing supply chains: a disruption risk management framework," Computers \& Operations Research, vol. 39, no. 2, pp. 350-357, 2012.

[16] Q. Sun, L. Jiang, and H. Xu, "Expectation-maximization algorithm of Gaussian mixture model for vehicle-commodity matching in logistics supply chain," Complexity, vol. 2021, Article ID 9305890, 2021.

[17] Q. Sun, L. Jiang, and H. Xu, "A double-layer combination algorithm for real-time information-sharing network design problem," Complexity, vol. 2021, Article ID 4856593, 2021.

[18] M. Chen, Y. Liu, Y. Song, and Q. Sun, "A contract coordination model of dual-channel delivery between UAVs and couriers considering the uncertainty of delivery for last mile," Discrete Dynamics in Nature and Society, vol. 2019, Article ID 6214359, 2019.

[19] M. Chen, Y. Liu, Y. Song, Q. Sun, and C. C. Cong, "Multimodal transport network optimization considering safety stock under real-time information," Discrete Dynamics in Nature and Society, vol. 2019, Article ID 5480135, 2019.

[20] Q. Sun, W.-G. Che, and H.-L. Wang, "Bayesian regularization BP neural network model for the stock price prediction," in Foundations and Applications of Intelligent Systems, pp. 521-531, Springer, Berlin, Germany, 2014.

[21] S. Ji and Q. Sun, "Low-carbon planning and design in B \& R logistics service: a case study of an E-commerce big data platform in China," Sustainability, vol. 9, no. 11, p. 2052, 2017.

[22] B. Xu, S. Zhu, J. Yu, C. Li, and Q. Sun, "Designing ETL processes to integrate multi-field digital information resources," in Proceedings of the 2017 2nd International Conference on Image, Vision and Computing (ICIVC), pp. 1053-1057, IEEE, Chengdu, China, June 2017.

[23] K. Deb, S. Agrawal, A. Pratap, and T. Meyarivan, “A fast elitist non-dominated sorting genetic algorithm for multi-objective optimization: NSGA-II," Parallel Problem Solving from Nature PPSN VI, vol. 1917, pp. 849-858, 2000.

[24] S. F. Ghannadpour and A. Zarrabi, "Multi-objective heterogeneous vehicle routing and scheduling problem with energy 
minimizing," Swarm and evolutionary computation, vol. 44, pp. 728-747, 2019.

[25] E. Zitzler and L. Thiele, "Multiobjective evolutionary algorithms: a comparative case study and the strength Pareto approach," IEEE Transactions on Evolutionary Computation, vol. 3, no. 4, pp. 257-271, 1999.

[26] E. Zitzler, K. Deb, and L. Thiele, "Comparison of multiobjective evolutionary algorithms: empirical results," Evolutionary Computation, vol. 8, no. 2, pp. 173-195, 2000. 